\title{
ClaimsKG: A Knowledge Graph of Fact-Checked Claims
}

\author{
Andon Tchechmedjiev ${ }^{1}$, Pavlos Fafalios ${ }^{2}$, Katarina Boland ${ }^{3}$, Malo Gasquet $^{5}$, \\ Matthäus Zloch ${ }^{3}$, Benjamin Zapilko ${ }^{3}$, Stefan Dietze ${ }^{3,4}$, Konstantin Todorov ${ }^{5}$ \\ ${ }^{1}$ LGI2P, IMT Mines-Ales, France \\ ${ }^{2}$ L3S Research Center, Leibniz University of Hanover, Germany \\ ${ }^{3}$ GESIS - Leibniz Institute for the Social Sciences, Germany \\ ${ }^{4}$ Heinrich-Heine-University Düsseldorf, Germany \\ ${ }^{5}$ LIRMM / University of Montpellier / CNRS, France \\ andon.tchechmedjiev@mines-ales.fr, fafalios@L3S.de, \\ malo.gasquet@etu.umontpellier.fr, \{katarina.boland, matthaeus.zloch, \\ benjamin.zapilko, stefan.dietze $@$ @esis.org, konstantin.todorov@lirmm.fr
}

\begin{abstract}
Various research areas at the intersection of computer and social sciences require a ground truth of contextualized claims labelled with their truth values in order to facilitate supervision, validation or reproducibility of approaches dealing, for example, with fact-checking or analysis of societal debates. So far, no reasonably large, up-to-date and queryable corpus of structured information about claims and related metadata is publicly available. In an attempt to fill this gap, we introduce ClaimsKG, a knowledge graph of fact-checked claims, which facilitates structured queries about their truth values, authors, dates, journalistic reviews and other kinds of metadata. ClaimsKG is generated through a semi-automated pipeline, which harvests data from popular fact-checking websites on a regular basis, annotates claims with related entities from DBpedia, and lifts the data to RDF using an RDF/S model that makes use of established vocabularies. In order to harmonise data originating from diverse fact-checking sites, we introduce normalised ratings as well as a simple claims coreference resolution strategy. The current knowledge graph, extensible to new information, consists of 28,383 claims published since 1996 , amounting to $6,606,032$ triples.
\end{abstract}

Keywords: Claims; Fact-checking; Societal debates; Knowledge Graphs

\section{Introduction}

The spread of controversies, biased discourse and falsehoods on the Web has become an increasingly important issue, from both a societal as well as a research perspective 1, 30. Recently, a wide range of interdisciplinary research directions are being explored in this broad area, which often rely on a ground truth of labelled claims. Such works include investigations into the spreading pattern of false claims on Twitter [30, pipelines for discovering the stance of claim-relevant 
(Web) documents [34, approaches for classifying sources of news, such as Web pages, domains, users or posts 7. 20], or research into fake news detection 27] and automatic fact-checking 12 . In all these cases, the availability of a labelled ground truth, consisting of claims, their corresponding metadata and, in particular, their truth values (or ratings), is essential in order to enable supervision of machine learning methods, reproduction and explainability of the results, and to facilitate fair evaluation and follow-up work. In addition, as documented by the aforementioned works, claims are usually not considered in isolation, but in a context. Thus, reproducing such research requires not only archiving claims and their truth values, but also their related documents, such as journalistic claim reviews, the associated entities and time-frames that can be linked to particular events, accounting in that way for the continuous evolution of Web content.

To our knowledge, no reasonably large and up-to-date corpus of structured information about claims and their context has been made publicly available. We attempt to fill this gap by introducing ClaimsKG, a knowledge graph (KG) of fact-checked claims, which facilitates structured queries about their truth values and other kinds of metadata, constructed and published following the W3C recommendations and best practices. In our context, we define a claim as a statement which has been reviewed by a fact-checking organisation in order to assess its truthfulness. ClaimsKG is generated through a semi-automated pipeline, which periodically harvests data from popular fact-checking websites. The claims and their reviews (articles written by fact-checkers that accompany a claim and explain its context and veracity judgement) are annotated with related entities from DBpedia, and all data are lifted into RDF using a dedicated RDF/S model (dubbed Claims), which is based on established vocabularies such as schema.org and NIF. In order to harmonise data originating from diverse fact-checking sites, we introduce a normalised truth ratings scheme, as well as a simple claim matching strategy. ClaimsKG enables advanced exploration and information discovery, e.g., via queries such as "find all false claims by D. Trump in 2017 that also mention the FBI", or "find the top 5 politicians per month involved in false claims", as well as exploitation of data from various sources via federated SPARQL queries, e.g., "retrieve all claims mentioning journalists". We also provide a Web interface for exploring the graph, enabling users from outside of the computer science community to retrieve information or sample data from our resource. The dataset, as of April 2019, consists of 28,383 claims published since 1996, amounting to 6,606,032 triples in our KG.

In summary, we provide (1) the Claims data model for representing factchecked claims and associated information, (2) an open-source pipeline for crawling and extracting data from fact-checking websites, and for lifting these data following the Claims model, (3) an openly available dynamic large-scale KG of claims and associated metadata, and (4) a Web interface for search and exploration of the resource. In the following section, we provide general information about the resource and links for access. We detail the KG generation process in Sect. 3. We introduce our Claims model in Sect. 4. while use-cases and queries 
are discussed in Sect. 5 along with an overview of the exploratory user interface. We review related work in Sect. 6 before concluding.

\section{ClaimsKG in a Nutshell}

ClaimsKG consists of data extracted from a number of fact-checking websites. To select the fact-checking websites, we relied on the International Fact-Checking Network's (IFCN) signatories list 1 admitting only sources considered by the fact-checking community as highly reputable. At this stage, we only consider information in English from six sources: africacheck.org, factscan.ca, politifact.com, snopes.com, checkyourfact.com, truthorfiction.com. Note that ClaimsKG is extensible to new websites, however, the information extraction process may vary from one website to another due to structural specificities of the sources (cf. Sect. 3). Each fact-checking article from these sources is parsed for extracting the text of the claim under review as well as useful related (meta)data including the author of the claim, the date the claim was uttered, its veracity label as well as keywords (tags describing topics) and links to related resources. Moreover, the text of the claim and of its review is annotated with Wikipedia/DBpedia entities mentioned in it. Key links related to ClaimsKG are given in Table 1. The KG is currently accessible from a Virtuoso triplestore with a SPARQL endpoint and downloadable as a Zenodo dump. All represented entities (claims, authors, etc.) are assigned resolvable identifiers following the W3C best practices (see Sect. 3 for an example). The dataset has a DCAT description and is released for free distribution under a Creative Commons Attribution-NonCommercial-ShareAlike 4.0 licence $\left.\right|^{2}$ The graph can be also accessed through ClaimsKG's official webpage, which displays detailed up-to-date statistics and a set of example SPARQL queries. All tools developed for the KG's creation are made available as open source on GitHub. Table 2 offers some general and per-source coverage statistics for the data, in particular the coverage of key properties. In order to account for emerging claims, the dataset is updated regularly (every 3-6 months).

Table 1: Key links to ClaimsKG's data and tools.

\begin{tabular}{l|l|}
\hline ClaimsKG website & https://data.gesis.org/claimskg/site \\
Dataset DOI & https://doi.org/10.5281/zenodo.2628745 \\
DCAT description & Included in the KG \\
Zenodo dump & https://zenodo.org/record/2628745 \\
SPARQL endpoint & https://data.gesis.org/claimskg/sparql \\
The Claims ontology & https://data.gesis.org/claimskg/site/\#model \\
\cline { 2 - 2 } Exploratory interface & https://data.gesis.org/claimskg/explorer \\
\cline { 2 - 2 } ClaimsKG pipeline source code & https://github.com/claimskg \\
\hline
\end{tabular}

1 https://ifcncodeofprinciples.poynter.org/signatories

2 https://creativecommons.org/licenses/by-nc-sa/4.0/ 
Table 2: Claim metadata coverage and statistics (as of April 2019)

\begin{tabular}{|c|c|c|c|c|c|c|c|}
\hline Property $\backslash$ Fact-checking website & Global & Snopes & Politifact & AfricaCheck & TruthOrFiction & CheckYourFact & FactScan \\
\hline Number of claims & 28,383 & 10,685 & 15,743 & 560 & 778 & 492 & 125 \\
\hline Claim text & $100 \%$ & $100 \%$ & $100 \%$ & $100 \%$ & $100 \%$ & $100 \%$ & $100 \%$ \\
\hline Claim author & $93.6 \%$ & $100 \%$ & $100 \%$ & $0.0 \%$ & $0.0 \%$ & $0.0 \%$ & $100 \%$ \\
\hline Claim date published & $92.1 \%$ & $96.3 \%$ & $100 \%$ & $0.0 \%$ & $0.0 \%$ & $0.0 \%$ & $98.4 \%$ \\
\hline Claim with references $(\geq 1)$ & $86.5 \%$ & $99.8 \%$ & $75.9 \%$ & $97.0 \%$ & $100 \%$ & $99.6 \%$ & $100 \%$ \\
\hline Claim with keywords $(\geq 1)$ & $93.8 \%$ & $95.4 \%$ & $100 \%$ & $99.5 \%$ & $0 \%$ & $0.0 \%$ & $100 \%$ \\
\hline Claim with entities $(\geq \overline{1})$ & $99.7 \%$ & $99.9 \%$ & $100 \%$ & $98.2 \%$ & $99.6 \%$ & $92.9 \%$ & $100 \%$ \\
\hline Claim review URL & $100 \%$ & $100 \%$ & $100 \%$ & $100 \%$ & $100 \%$ & $100 \%$ & $100 \%$ \\
\hline Claim review title & $100 \%$ & $100 \%$ & $100 \%$ & $100 \%$ & $100 \%$ & $100 \%$ & $100 \%$ \\
\hline Claim review author & $100 \%$ & $100 \%$ & $100 \%$ & $100 \%$ & $100 \%$ & $100 \%$ & $100 \%$ \\
\hline Claim review date published & $100 \%$ & $100 \%$ & $100 \%$ & $100 \%$ & $0 \%$ & $100 \%$ & $100 \%$ \\
\hline Claim review language & $100 \%$ & $100 \%$ & $100 \%$ & $100 \%$ & $100 \%$ & $100 \%$ & $100 \%$ \\
\hline Claim review with entities $(\geq 1)$ & $66.9 \%$ & $74.1 \%$ & $58.7 \%$ & $80.2 \%$ & $76.0 \%$ & $97.0 \%$ & $96.0 \%$ \\
\hline Claim rating & $100 \%$ & $100 \%$ & $100 \%$ & $100 \%$ & $100 \%$ & $100 \%$ & $100 \%$ \\
\hline Exact claim matches & 87 & 38 & 49 & 0 & 0 & 0 & 0 \\
\hline True claims & 3,725 & 1,311 & 2,255 & 60 & 97 & 0 & 2 \\
\hline False claims & 11,068 & 6,002 & 4,663 & 209 & 191 & 0 & 3 \\
\hline Mixture claims & 10,420 & 1,798 & 8,564 & 0 & 56 & 2 & 0 \\
\hline Other claims & 3,170 & 1,574 & 261 & 291 & 434 & 490 & 120 \\
\hline
\end{tabular}

\section{Generating ClaimsKG}

ClaimsKG is built through a pipeline, which periodically crawls popular factchecking sites, normalises ratings and entity mentions, reconciles identical claims, and lifts the data onto the specifically developed Claims model, described in Sect. 4. Hereafter, we detail the technical steps of the pipeline, summarised in Fig. 1. Links to its open-source components are given in Table 1.

Extracting claims and metadata. The Claims extractor crawls the identified fact-checking websites and collects the information in a JSON or Microdata format to consolidate a large multi-sourced data set (as a CSV file). The collected data consist mainly of: (a) the textual statement of the claim; (b) its truth-value or rating (both the normalised and the original one); (c) a link to the claim review from the fact-checking website; (d) the references cited in the claim reviews; (e) the entities extracted from the text of the claim and from the review body; (f) the author of the claim and the author of the review; (g) the date of publication of the claim and that of the review; (h) the title of the review article; (i) a set of keywords extracted from the websites acting as topics (e.g. "abortion").

Note that the extraction process is tailored individually to the structure of each of the different fact-checking websites, resulting in a set of website-specific extractors. The statistics generated at each run of the pipeline (globally and per domain) allow to monitor the "health" of the extracted data by detecting potential issues that may be related to changes of the structures of the respective fact-checking websites that may have occurred between two runs of the pipeline.

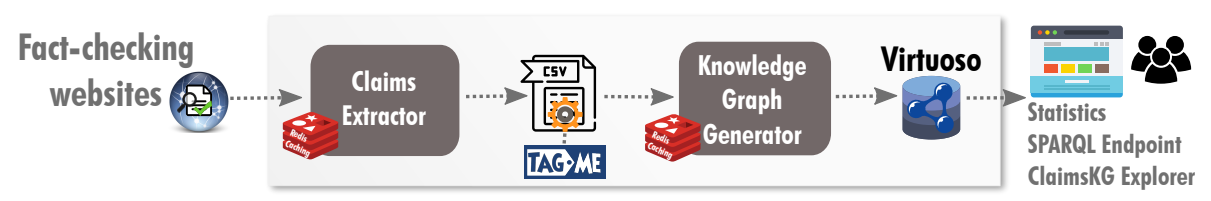

Fig. 1: Overall architecture of the ClaimsKG pipeline. 
Entity annotation of the claims. We annotate the entities (e.g., names of persons, organisations, locations, etc.) mentioned in the texts of the claims and their reviews using the TagMe tool 8]. TagMe allows the automatic identification of entities in a text and their linking to a Wikipedia page and a DBpedia URI. It is known to achieve particularly good results when annotating short texts, which is the case for the statements in this domain, although we also annotate the body of the claim reviews. We run a local version of TagMe, allowing us to update the database regularly, using the latest available dump of Wikipedia (October 2018). We performed all the annotations using the optimal parameters described in 8. We evaluated our updated TagMe model on the YAGO CONLL-TestB ground truth dataset 14 and obtained an accuracy of $75.5 \%$, which is in line with state of the art performance reported for TagMe ${ }^{3}$

Normalization of ratings. Each of the fact-checking websites has its own labels describing the truthfulness of the claims, with different discrete textual values of ratings. While some sites have a controlled vocabulary of possible truth values, others apply an open-ended rating schema. For example, Truth or Fiction has a large number of non-uniform labels, such as "truth \& misleading" or "reported as fiction". In order to harmonize our dataset, alongside the original ratings, we also provide a normalized rating score, applied across all claims contained in the dataset. For each of the sources, we summarised the distribution of rating values and then assigned them to a conservative and coarser-grained set of labels that correspond to the least common denominator between all the classifications of the individual sites. Given the varied rating schemes, where individual labels often are hard to objectively apply or interpret, we opted for a simple rating scheme consisting of four basic categories (TRUE, FALSE, MIXTURE, OTHER) that can be mapped to existing rating schemes ${ }^{4}$ The two extreme cases of a claim being proven true or false are captured by TRUE and FALSE, while MIXTURE characterises something on a truth scale or that holds both a degree of truth and a degree of falsehood. For anything that does not fall into this spectrum, we chose OTHER as a fallback. While the TRUE/FALSE ratings are straightforward, MIXTURE conflates a very large number of possible truth values, as diverse as "downplayed" or "mostly true". For OTHER, we have rating names such as "half-flip", "scam" or "research in progress".

Lifting and Serialisation. We created a Python 3.6 script to read the extracted claims as a CSV file in the extraction step, and then create the corresponding KG following the data model described in Sect. 4. We used the rdflib library to create the model and an abstract RDF graph to then serialize it in one of the many supported formats. All the caching needs of the generation process are met with a Redis server. We generate unique URI identifiers as UUIDs based on an one-way hash of key attributes for each instance. For example, the dereferenceable URI http://data.gesis.org/claimskg/creative_work/ 5f7e8c65-3d8b-57da-bab9-eb3a373bd2ab is created for the claim in https://

3 http://nlpprogress.com/english/entity_linking.html

${ }^{4}$ We provide full correspondence tables here: https://goo.gl/Ykus98 
WWW. snopes.com/fact-check/was-megyn-kelly-fired-from-nbc/ The triplification package is made available under an open-source licence on GitHub (along with documentation and usage examples) and will be updated regularly with the latest improvements (link given in Table 1).

Handling simple claim coreferences. A certain number of identical claims is present within the websites, published at different dates, with possibly varying reviews. For example, the same claim published at a later date than the original publication will have an updated review. For this reason, instead of fusing these claims, we have opted for establishing owl: sameAs links among them. We implemented a simple approach to identify these claims, which aims to ensure $100 \%$ precision of the discovered links (exact matches). We normalise the text of the claims and the text of the claim titles only (lowercase; remove all quote characters and certain stop-words, such as "said" and "claimed") and then apply an identity string similarity measure on these texts. This resulted in 38 owl: sameAs links on claims from Snopes and 49 from Politifact.

\section{The Claims Data Model}

Our data model, depicted in Fig. 2, exploits terms from established vocabularies, specifically schema.org, NLP Interchange Format (NIF) $5^{5}$ and Internationalization Tag Set (ITS) ${ }^{6}$ The selection of the vocabularies was based on the following objectives: i) avoiding schema violations, ii) enabling data interoperability through term reuse, iii) having stable identifiers, persistent hosting and open license, iv) being supported by a community, v) being extensible (ability to easily extend ClaimsKG with more data).

The core elements of our model are the claim and the claim review. To represent them, we make use of schema.org. Following Google's suggestion for Web markup of claims 7 a claim is of type schema:CreativeWork and a claim review of type schema:ClaimReview. An instance of schema:ClaimReview is connected to an instance of schema:CreativeWork through the property schema:itemReviewed. A claim is associated with the actual text of the claim, a date (when the claim was uttered), an author (who uttered the claim), as well as with keywords (tags related to the claim acting as topics) and one or more citations (URLs of related resources, e.g., a tweet or a video). Since there might be many instances of the same claim coming from the same or different fact-checking sites, two claims can be connected through a owl: sameAs property.

A claim review is associated with metadata, in particular its author, its publication date, its language, its URL (pointing to the full text of the review), its full text (optionally, according to copyright restrictions), as well as with a title and one or more truthfulness assessments (ratings). The assessment is of

\footnotetext{
5 https://persistence.uni-leipzig.org/nlp2rdf/ontologies/nif-core/ nif-core.html

o https://www.w3.org/TR/its20/

7 https://developers.google.com/search/docs/data-types/factcheck
} 


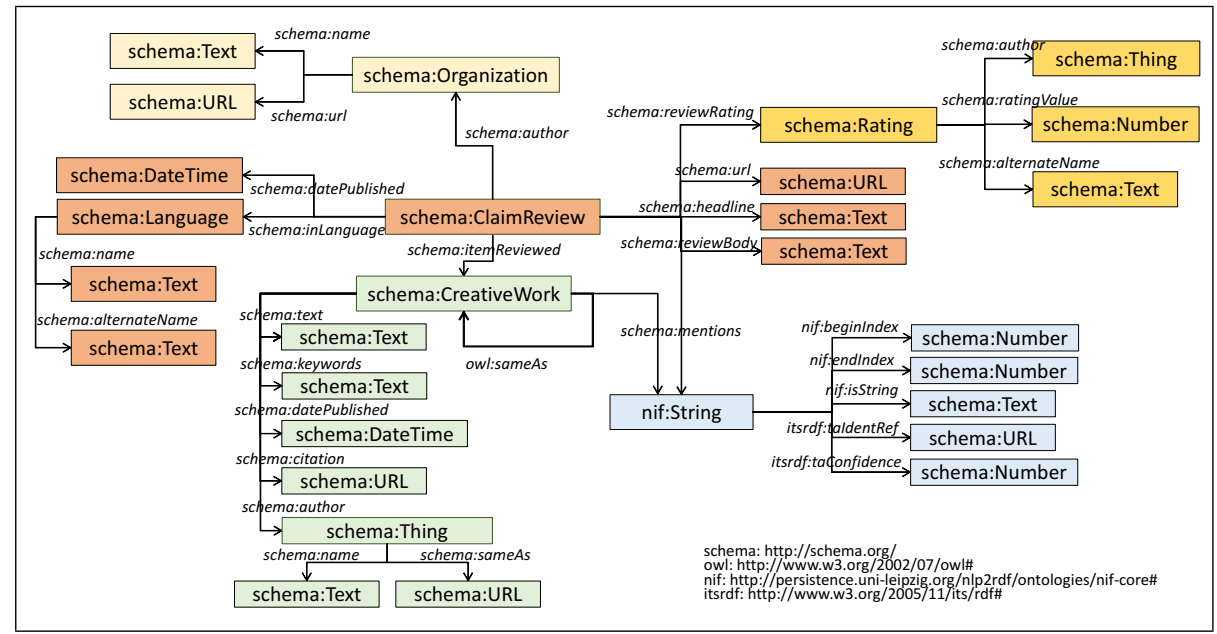

Fig. 2: The Claims data model.

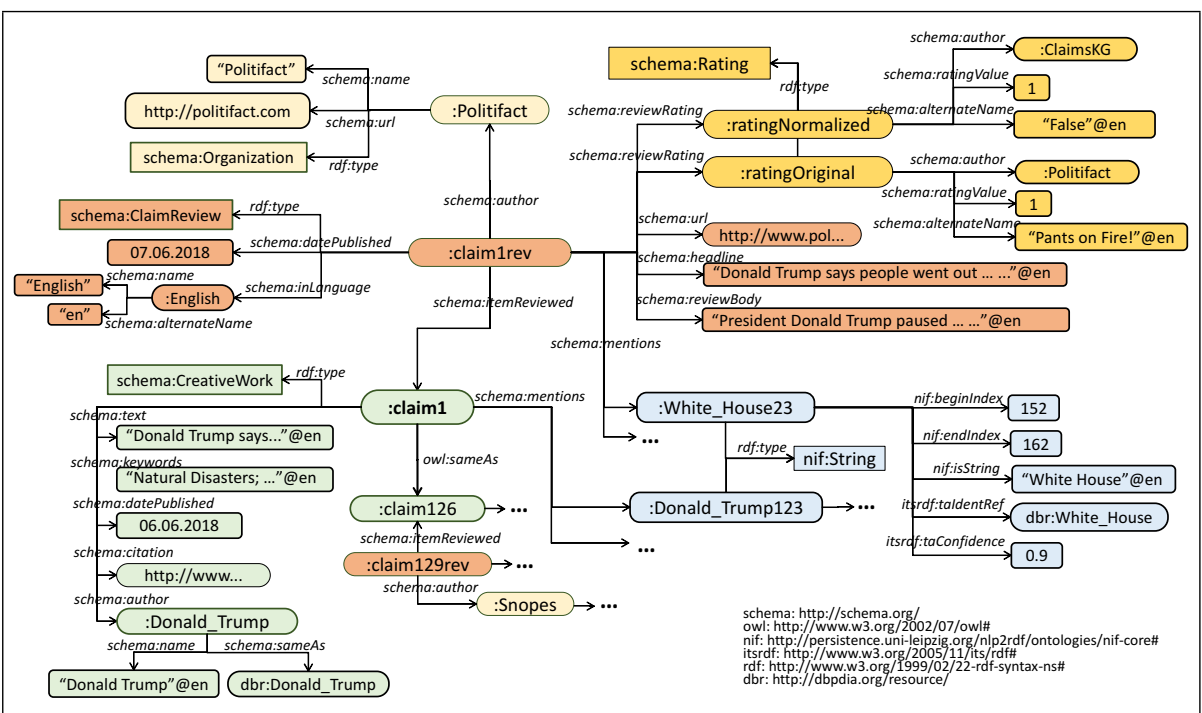

Fig. 3: Instantiation of the Claims model for a claim sourced from Politifact made by Donald Trump on June 6, 2018. 
type schema:Rating and is connected with the review through the property schema:reviewRating. A rating is represented through three properties: author (who provides the rating), rating value (a number in a pre-specified range, e.g., 1 to 5), and alternate name (a textual label of the rating value, e.g., "false"). The author property allows to provide more than one ratings for the same instance of a claim review.

Both a claim and a claim review can be associated with one or more entity mentions, i.e., names of entities mentioned in the short text of the claim or in the claim review. To describe this information, we make use of the NIF and ITS vocabularies, which provide classes and properties to describe the result of natural language processing tools applied on texts or documents. An instance of an entity mention is described through five properties: nif : beginIndex (starting position in the text), nif : endIndex (ending position in the text), nif : isString (a word or sequence of words representing the entity), itsrdf : taIdentRef (the identity of the mentioned entity), and itsrdf:taConfidence (the confidence that the entity has been disambiguated correctly). Depending on the specific requirements with respect to precision and recall, data consumers can select suitable confidence ranges to consider when querying the data.

Fig. 3 depicts an example of a claim review by Politifact for a claim made by Donald Trump on June 6,2018 ${ }^{8}$ We notice that there are two instances of schema:Rating, one for the original rating by Politifact and one for the normalized rating provided by our KG. Apart from metadata information, we also see that the review mentions the entity name "White House", which probably (with confidence 0.9/1.0) corresponds to the official residence of the US President.

\section{Use Cases and Exploitation}

Use-cases and queries. The publication of structured data about a large collection of claims allows the uncovering of explicit and implicit relations between claims, entities, and sources. A number of existing fact-checking applications rely on linking claims to fact-checked statements in a database (e.g. https://fullfact.org/automated/, 32 35]). By combining claims and ratings from multiple portals and providing a unified structure, ClaimsKG facilitates these efforts. Moreover, the data can be used to enable supervision of machine learning models to support the advancement of automatic fact-checking algorithms [13 20. In addition to supplying a large number of claims, ClaimsKG enables advanced, entity-centric search, exploration and information discovery, by exploiting data from various sources via federated SPARQL queries. This allows us, for example, to query entities belonging to a specific group (e.g., politicians or journalists) and create complex queries using both the claims metadata and the extracted entities. We provide different examples on our website (see Table 1 for a link). By exploiting the claim metadata and extracted entities, we can run complex queries that combine different types of information. The query in Fig. 4

8 http://www.politifact.com/texas/statements/2018/jun/07/donald-trump/ donald-trump-says-people-went-out-their-boats-watc/ 
requests all false claims of 2017 mentioning Donald Trump and Climate change. For each claim, the query returns its text, date, as well as the URL of its review by a fact-checking site. The query returns the following claim: Donald Trump signed an executive order naming climate change as a threat 'both to the economy and national security' (2017-02-01). In a similar way, we can generate a sample of claims based on certain criteria and use it in other tasks, e.g., for evaluation or training by automated fact-checking approaches 13,20 . Such a sample can be easily produced through a concise SPARQL query over ClaimsKG.

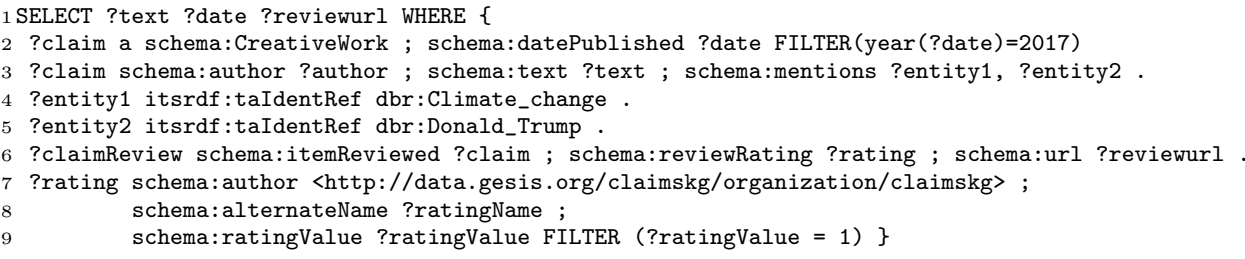

Fig. 4: SPARQL query requesting false claims of 2017 mentioning both Donald Trump and Climate change.

Going beyond the computer science domain, ClaimsKG can be a valuable resource supporting (computational) social scientific research investigating, for example, societal debates and agenda-setting. Agenda-setting theory refers to the influence of mass media on the public's focus of attention [22]. While firstlevel agenda-setting relates to inserting topics, events or entities into the public discourse, thereby regulating societal priorities, second-level agenda-setting is about increasing the salience of specific features or attributes of entities in the discourse. This is also referred to as frame-setting. With the web evolving into a platform where every citizen may become a publisher, express their views and reach out to a large audience, citizens are now able to play a more active role in influencing the public discourse 3]. Online debates about political issues typically exhibit the pattern of a few dominant ideological positions emerging, with different groups expressing different viewpoints and often referring to a disparate set of information sources 24] which, in turn, may focus on different attributes and frames for a given topic. Using ClaimsKG, an exploratory search on a topic and related entities may be performed in order to gain insights on relevant viewpoints, attributes and actors. Also, the KG allows the tracking of differences over time and in relation to specific events, and the relation of views of specific actors to ideological positions.

To illustrate, consider the 2012 incident of the neighbourhood watch coordinator George Zimmerman shooting 17-year-old African-American high school student Trayvon Martin, the incident that later gave rise to the Black Lives Matter movement [15]. The query given in Fig. 5 retrieves all claims mentioning Trayvon Martin or George Zimmerman, yielding 68 claims in total with 8 claims rated true, 33 false, and 24 mixture. The distribution of truth values hints at this being a highly controversial topic with potentially highly polarized viewpoints. Central to the debate is the aspect of racism; some framing the in- 
cident as an example of racist violence against black people 9 some seeing race as an overemphasized point in the Zimmerman tria ${ }^{10}$, and others framing the Black Lives Matter debate as racist against white people ${ }^{11}$ An entity frequently mentioned in these claims is the "stand your ground" law. The query in Fig. 6 retrieves other entities connected to it revealing a strong association to the Trayvon Martin case.

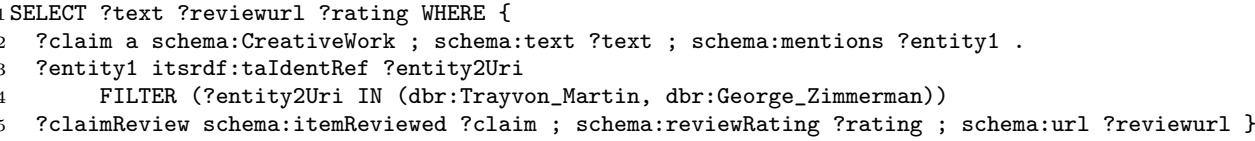

Fig. 5: SPARQL query requesting all claims mentioning Trayvon Martin or George Zimmerman.

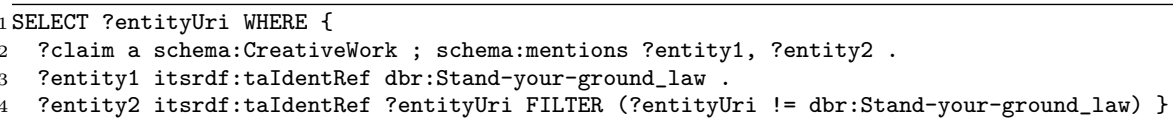

Fig. 6: SPARQL query requesting entities mentioned in claims together with Stand your ground law.

SELECT year(?date) as ?year count(?claim) as ?num WHERE

2 ?claim a schema:CreativeWork ; schema: datePublished ?date FILTER (year(?date)>=2012)

3 ?claim schema:author ?author ; schema:text ?text ; schema:mentions ?entity .

4 ?entity itsrdf:taIdentRef dbr:Black_Lives_Matter \} GROUP BY year(?date) ORDER BY year(?date)

Fig. 7: SPARQL query requesting the number of claims mentioning Black Lives Matter by year.

The query in Fig. 7 illustrates the tracking of changes over time and the discovery of important events. While for 2015, 2017, 2018 and 2019 maximum three claims per year mentioning the entity Black Lives Matter are found, there is a striking peak in 2016 with 17 mentions. This aligns with the incident of law enforcement officers being shot during a Black Lives Matter protest march in July 2016 which reopened a heated debate about the movement. In fact, analysis of the respective claims reveals the emergence of frames attributing violent and disruptive behaviour to the Black Lives Matter movement ${ }^{12}$ Note that the discussed scenarios represent only starting points for initiating further analyses.

\footnotetext{
$9 \longdiv { \text { https://www } . p o l i t i f a c t . c o m / f l o r i d a / s t a t e m e n t s / 2 0 1 3 / j u l / 2 4 / ~ }$ jesse-jackson/homicides-blacks-have-tripled-stand-your-ground-wa/ 
ClaimsKG Explorer. In order to facilitate data access for researchers from outside of the computer science domain, like journalists and sociologists, we provide ClaimsKG Explorer (link in Table 1), a user-friendly, Web-based interface to query and explore ClaimsKG. The application sends HTTP requests to the ClaimsKG SPARQL endpoint and provides information through a Web user interface. The users are given the possibility to filter their search space with respect to a number of facets (Fig. 8a): entities contained in the text of the claim or its review, keywords (topics related to the claims), truth ratings (the normalised ones), time frame of interest, authors of claims, sources (fact-checking websites), and languages (currently only English; work in progress will incorporate non-English websites). After clicking on the CLAIMS SEARCH button, the user is provided a list of clickable claims ordered by their date of publication (most recent ones on top), as shown in Fig. 8b. The search result corresponding to the selected criteria can be exported as a CSV or an RDF file and reused in a particular scenario by clicking on the EXPORT button on the results page. Finally, cicking on a particular claim, the user can access all the information related to that claim, like the review article, the entities mentioned in the claim or the review text, the references, and the keywords.

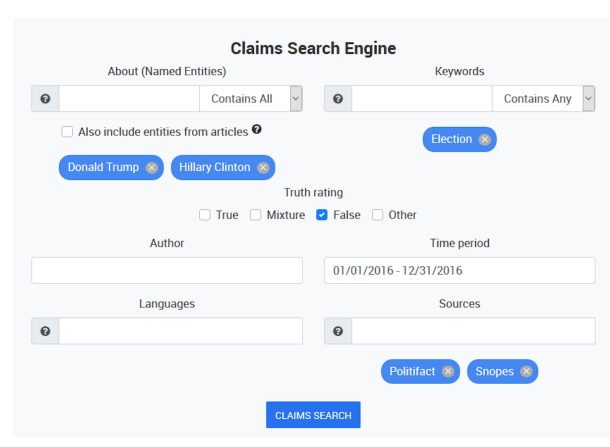

(a) The Explorer's search engine.

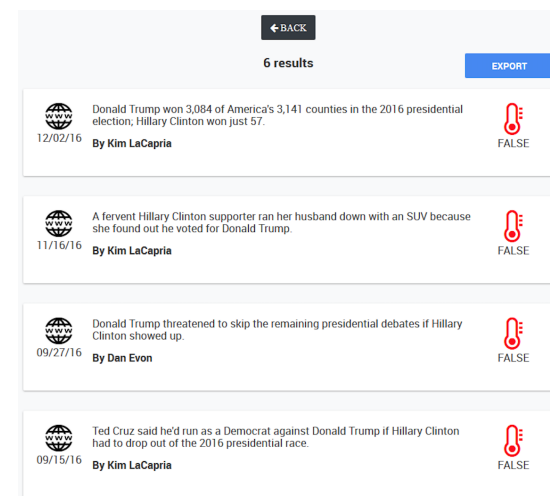

(b) Results

Fig. 8: The ClaimsKG Explorer user interface

\section{Related Work}

As outlined above, ground truth data in the form of labelled and contextualised claims is necessary for a number of interdisciplinary research problems. Among the most prominent use-case scenarios is the task of automatic factchecking, which has been of growing interest for the AI community. A number of approaches have been proposed to extract check-worthy pieces of information from text 12 and to further assess their veracity automatically [31. The majority of these approaches can be classified either as reference or machine 
learning approaches. The former model claims computationally to achieve structured representations [4, 23 $29,32,35$, allowing for their comparison to certified facts contained in knowledge bases or for the application of graph mining techniques on these bases. The latter rely on data in the form of labelled claims in order to train and apply machine learning models. The current section provides an overview of datasets for training and/or evaluation covering these two families of approaches. We categorise these datasets according to the process of their collection.

Extracting gold standard from web sources. Alongside ClaimsKG, a number of approaches rely on extracting data from fact-checking websites, allowing the collection of large number of claims together with veracity annotations of high quality that stem from serious journalistic work. One of the first initiatives in that field is introduced in [28]. The data extraction process is manual, resulting in merely 221 statements collected from Channel 4 and PolitiFact. Since the two sources do not apply the same rating scale, similarly to our approach, the authors map the two respective sets of labels to a common scale consisting of five categories: "true", "mostly true", "half true", "mostly false" and "false". The Liar benchmark 33 collects $12.8 \mathrm{~K}$ Politifact claims over 10 years. Additional information is stored regarding the speaker, the context, the label and the justification. The fact-checking approach presented in [21 relies on a data set of approximately $10 \mathrm{~K}$ claims, also crawled from Politifact in 2016, while 16] and 20 have collected, respectively, $1 \mathrm{~K}$ and $5 \mathrm{~K}$ claims from Snopes. Wikipedia's lists of proven hoaxes $s^{13}$ and fictitious peopl ${ }^{14}$ have been used in 19,20 in order to generate ground truth labels, resulting in 157 claims labelled as "fake". In parallel, the authors also collect around $4.8 \mathrm{~K}$ labelled claims from Snopes, published before Faburary 2016. The Clef-2018 challenge includes the fact-checking task Check that! [2]. The benchmark data (150 claims) consists of sentences collected from debates from the 2016 US Presidential Election Campaign, as well as from other political speeches during and after the campaign. Evaluations are obtained from FactCheck.org articles resulting in labels of the kind "factually true", "half-true", or "false".

The Emergent data set 15 results from collecting claims from various web sources, such as Politifact, Snopes and Twitter accounts such as @Hoaxalizer, particularly dedicated to rumors and hoaxes [9]. The data set contains 300 claims with three types of annotations ("true", "false", "unverified") provided by journalists. A large scale collection of tweet news (126K stories) labelled on the basis of significant degree of agreement among several fact-checking sites (Snopes, Politifact, Factcheck, Truth-or-Fiction, Hoax-slayer and Urbanlegends) is used in 31. As a result, a sample of news stories assigned one of three possible labels ("true", "false" or "mixed") is created. However, the data is only available upon request for the purposes of reproducing the reported experiments.

13 https://en.wikipedia.org/wiki/Listofhoaxes\#Provenhoaxes

14 https://en.wikipedia.org/wiki/List_of_fictitious_people

15 http://www.emergent.info 
Crowdsourced or manually annotated data sets. Crowdsourcing techniques allow for the extraction and labelling of relatively large sets of claims, although some care should be taken to ensure the reliability of the data harvested. The Open Domain Deception Dataset contains "freely contributed truths and lies" [18]. By using the Amazon Mechanical Turk, each worker has been asked to freely formulate seven one-sentence truths and lies. After cleansing the collection, the final dataset consists of $7.2 \mathrm{~K}$ sentences provided by 512 unique contributors, for whom demographic data is also collected and made available. The SemEval'17 challenge dataset contains $5.5 \mathrm{~K}$ crowdsourced annotated claims [1], while the FEVER dataset, with $185 \mathrm{~K}$ entries, extracts claims from Wikipedia. Semanticspreserving sentence altering techniques are then applied and the resulting claims are annotated by the crowd 25]. Finally, the approach in 17 relies on a dataset of 250 manually annotated claims.

Automatic annotations. Fact verification methods can be applied in order to construct automatically ground truth datasets for fact-checking. An example is the fake-news dataset ${ }^{16}$ containing text and metadata scraped from 244 websites that have been identified as untrustworthy by the BS Detector Chrome Extension tool ${ }^{17}$ This approach has the risk that the imperfections of the verification system are propagated onto the annotated data.

Knowledge graphs for fact-checking. Finally, we focus on KGs that have been used in a number of fact verification approaches from the reference group. Usually, a statement is modelled as a triple and is verified on the basis of properties of the paths involving elements of that triple in existing KGs, considered as ground truth, such as DBpedia 4, 10,23. The Knowledge Vault 6 and the KnowMore 37] resources, or the Voldemort KG [26], rely on structured markup annotations in order to match them to established KGs or perform graph completion. This process implies the verification of the truthfulness of statements and the production of reliable factual information that can be used as ground truth.

Positioning. ClaimsKG is entirely based on data from a number of established fact-checking websites and therefore falls into the first category of datasets presented above. With its more than $28 \mathrm{~K}$ claims, it is, to our knowledge, the largest resource of structured fact-checking information so far made available but also one archiving the largest spectrum of metadata categories. The open-source tools for its regeneration and update that we provide will allow for it to grow in size over time. In contrast to existing approaches, we model claims by the help of an $\mathrm{RDF} / \mathrm{S}$ data model specifically designed for that purpose, fostering re-usability and extensibility. As compared to KG-based reference approaches, by dynamically collecting data from fact-checking websites, we focus on information of particular interest for the verification of newly emerging statements that are not available in Wikipedia or established KGs. ClaimsKG can be used as both training and evaluation data, allowing users (researchers in computer science or

16 https://www .kaggle.com/mrisdal/fake-news

17 http://bsdetector.tech 
computational sociology, for example) to compile thematic samples of it with the help of structured queries, or by using the web application (cf. Sect. 5). Beyond the purposes of fact-checking, this is expected to foster research and data-driven studies in different areas of social and computational social science, as discussed in our use-case scenarios.

\section{Conclusion and Future Work}

We have introduced ClaimsKG, a knowledge graph of fact-checked claims, which facilitates structured queries of related metadata, such as their truth values, authors or time of release. ClaimsKG is generated through a semi-automated pipeline, which harvests data from popular fact-checking sites on a regular basis, lifts data into a specifically developed for that purpose model, and annotates claims with related entities from DBpedia. The KG is expected to provide support to research in the areas of fact-checking, stance detection and multiple topics related to the analysis of societal debates, where a quality ground truth of labelled claims is required in order to facilitate supervision, validation or reproducibility of research methods.

There are several limitations of the current KG that are the focus of ongoing and short-term efforts. The development of an advanced claim matching approach and its evaluation is among them. We are working on building a goldstandard dataset of claim-pairs, annotated with respect to different relatedness categories, in order to evaluate the process and to provide training data to finetune state-of-the-art deep language modelling approaches such as BERT [5] to our matching task. We also intend to extend the content of our graph to other fact-checking websites and languages, enabling multi- and cross-lingual information retrieval and approaches for fact verification. With respect to augmenting ClaimsKG with additional claims, we also intend to harvest semi-structured schema.org markup of claims from Web pages by exploiting data fusion pipelines developed as part of prior work [36]. Regarding the exploratory Web interface, in the future we aim to support the execution of federated queries that integrate information from external KGs like DBpedia, as well as the inclusion of a statistical observatory allowing us to extract distributions and correlations of different entities, topics and claims.

\section{Acknowledgements}

We thank Vinicius Woloszyn for providing support on the first step of the pipeline (claim extraction), as well as Josselin Alezot, Imran Meghazi and Elisa Gueneau for their work on the Web interface. We thank all fact-checking sites and the fact-checkers community for the laborious work of manual claim verification.

\section{References}

1. Allcott, H., Gentzkow, M.: Social media and fake news in the 2016 election. Journal of Economic Perspectives 31(2), 211-36 (2017) 
2. Barrón-Cedeño, A., Elsayed, T., Suwaileh, R., Màrquez, L., Atanasova, P., Zaghouani, W., Kyuchukov, S., Da San Martino, G., Nakov, P.: Overview of the clef-2018 checkthat! lab on automatic identification and verification of political claims, task 2: Factuality. In: CLEF. CEUR-WS (2018)

3. Bennett, W.L., Pfetsch, B.: Rethinking Political Communication in a Time of Disrupted Public Spheres. J. of Communication 68(2), 243-253 (04 2018). https://doi.org/10.1093/joc/jqx017, https://doi.org/10.1093/joc/jqx017

4. Ciampaglia, G.L., Shiralkar, P., Rocha, L.M., Bollen, J., Menczer, F., Flammini, A.: Computational fact checking from knowledge networks. PloS one (2015)

5. Devlin, J., Chang, M.W., Lee, K., Toutanova, K.: BERT: Pre-training of Deep Bidirectional Transformers for Language Understanding. ArXiv e-prints, cs.CL 1810.04805 (2018)

6. Dong, X., Gabrilovich, E., Heitz, G., Horn, W., Lao, N., Murphy, K., Strohmann, T., Sun, S., Zhang, W.: Knowledge vault: A web-scale approach to probabilistic knowledge fusion. In: ACM SIGKDD. pp. 601-610. ACM (2014)

7. Esteves, D., Reddy, A.J., Chawla, P., Lehmann, J.: Belittling the source: Trustworthiness indicators to obfuscate fake news on the web. In: 1st Workshop on Fact Extraction and VERification (FEVER). pp. 50-59 (2018)

8. Ferragina, P., Scaiella, U.: Tagme: On-the-fly annotation of short text fragments (by wikipedia entities). In: ACM ICIKM. pp. 1625-1628. ACM (2010)

9. Ferreira, W., Vlachos, A.: Emergent: a novel data-set for stance classification. In: NAACL-HLT. pp. 1163-1168 (2016)

10. Gerber, D., Esteves, D., Lehmann, J., Bühmann, L., Usbeck, R., Ngomo, A.C.N., Speck, R.: Defactotemporal and multilingual deep fact validation. Web Semantics: Science, Services and Agents on the World Wide Web 35, 85-101 (2015)

11. Gorrell, G., Bontcheva, K., Derczynski, L., Kochkina, E., Liakata, M., Zubiaga, A.: Rumoureval 2019: Determining rumour veracity and support for rumours. Semantic Evaluation pp. 60-67 (2017)

12. Hassan, N., Adair, B., Hamilton, J.T., Li, C., Tremayne, M., Yang, J., Yu, C.: The quest to automate fact-checking. world (2015)

13. Hassan, N., Arslan, F., Li, C., Tremayne, M.: Toward automated fact-checking: Detecting check-worthy factual claims by claimbuster. In: ACM SIGKDD. pp. 1803-1812. ACM (2017)

14. Hoffart, J., Yosef, M.A., Bordino, I., Fürstenau, H., Pinkal, M., Spaniol, M., Taneva, B., Thater, S., Weikum, G.: Robust disambiguation of named entities in text. In: EMNLP. pp. 782-792. ACL, Stroudsburg, PA, USA (2011), http: //dl.acm.org/citation. cfm?id=2145432.2145521

15. Hon, L.: Social media framing within the million hoodies movement for justice. Public Relations Review $42 \quad\left(\begin{array}{ll}12 & 2015\end{array}\right)$. https://doi.org/10.1016/j.pubrev.2015.11.013

16. Ma, J., Gao, W., Mitra, P., Kwon, S., Jansen, B.J., Wong, K.F., Cha, M.: Detecting rumors from microblogs with recurrent neural networks. In: IJCAI. pp. 3818-3824 (2016)

17. Mihaylova, T., Nakov, P., Marquez, L., Barron-Cedeno, A., Mohtarami, M., Karadzhov, G., Glass, J.: Fact checking in community forums. AAAI pp. 879-886 (2018)

18. Pérez-Rosas, V., Mihalcea, R.: Experiments in open domain deception detection. In: CEMNLP. pp. 1120-1125 (2015)

19. Popat, K., Mukherjee, S., Strötgen, J., Weikum, G.: Credibility assessment of textual claims on the web. In: ACM ICIKM. pp. 2173-2178. ACM (2016) 
20. Popat, K., Mukherjee, S., Strötgen, J., Weikum, G.: Where the truth lies: Explaining the credibility of emerging claims on the web and social media. In: WWW. pp. 1003-1012 (2017)

21. Rashkin, H., Choi, E., Jang, J.Y., Volkova, S., Choi, Y.: Truth of varying shades: Analyzing language in fake news and political fact-checking. In: EMNLP. pp. 29312937 (2017)

22. Scheufele, D.A.: Agenda-setting, priming, and framing revisited: Another look at cognitive effects of political communication. Mass Communication and Society 3(2-3), 297-316 (2000). https://doi.org/10.1207/S15327825MCS0323_07, https: //doi.org/10.1207/S15327825MCS0323_07

23. Shiralkar, P., Flammini, A., Menczer, F., Ciampaglia, G.L.: Finding streams in knowledge graphs to support fact checking. In: ICDM. pp. 859-864. IEEE (2017)

24. Smith, M., Shneiderman, B., Rainie, L., Himelboim, I.: Mapping twitter topic networks: From polarized crowds to community clusters (02 2014)

25. Thorne, J., Vlachos, A., Christodoulopoulos, C., Mittal, A.: Fever: a large-scale dataset for fact extraction and verification. NAACL-HLT pp. 809-819 (2018)

26. Tonon, A., Felder, V., Difallah, D.E., Cudré-Mauroux, P.: Voldemortkg: Mapping schema. org and web entities to linked open data. In: ISWC. pp. 220-228. Springer (2016)

27. Tschiatschek, S., Singla, A., Gomez Rodriguez, M., Merchant, A., Krause, A.: Fake news detection in social networks via crowd signals. In: WWW. pp. 517-524 (2018)

28. Vlachos, A., Riedel, S.: Fact checking: Task definition and dataset construction. In: Language Technologies and Comp. Social Science. pp. 18-22 (2014)

29. Vlachos, A., Riedel, S.: Identification and verification of simple claims about statistical properties. In: CEMNLP. pp. 2596-2601. ACL (2015)

30. Vosoughi, S., Roy, D., Aral, S.: The spread of true and false news online. Science 359(6380), 1146-1151 (2018)

31. Vosoughi, S., Roy, D., Aral, S.: The spread of true and false news online. Science 359(6380), 1146-1151 (2018)

32. Walenz, B., Wu, Y., Song, S., Sonmez, E., Wu, E., Wu, K., Agarwal, P.K., Yang, J., Hassan, N., Sultana, A., et al.: Finding, monitoring, and checking claims computationally based on structured data. In: Computation+Journalism (2014)

33. Wang, W.Y.: Liar, liar pants on fire: A new benchmark dataset for fake news detection. AMACL pp. 422-426 (2017)

34. Wang, X., Yu, C., Baumgartner, S., Korn, F.: Relevant document discovery for fact-checking articles. In: WWW. pp. 525-533 (2018)

35. Wu, Y., Agarwal, P.K., Li, C., Yang, J., Yu, C.: Toward computational factchecking. Proceedings of the VLDB Endowment 7(7), 589-600 (2014)

36. Yu, R., Gadiraju, U., Fetahu, B., Lehmberg, O., Ritze, D., Dietze, S.: Knowmore knowledge base augmentation with structured web markup. Semantic Web Journal, IOS Press (2019), http://www.semantic-web-journal.net/content/ knowmore-knowledge-base-augmentation-structured-web-markup-1

37. Yua, R., Gadirajua, U., Fetahua, B., Lehmbergb, O., Ritzeb, D., Dietzea, S.: Knowmore-knowledge base augmentation with structured web markup. Semantic Web J., IOS Press (2017) 